\title{
A comparative study between codes of spectrum for a single degree of freedom (sdof) system in two different hazardous regions.
}

\begin{abstract}
Since in structure and earthquake engineering design of structures using response spectrum method (RSM) is very important, this study has been performed for a single degree of freedom (SDOF) system. Firstly the concept and the way of construction of response spectrum has been briefly explained. Then the records of some strong earthquakes in USA and Iran as two hazardous regions have been plotted, after that selected response spectrums (RS) of each country has been compared with each other and finally with standard response code of its own country. It was concluded:1) For a given ground motion the response of a SDOF system only depends on its natural vibration period $(\mathrm{T})$ and damping ratio( $(\zeta)$.2) When the effective damping ratio of a structure increases, its dynamic responses will decrease which demands the use of higher value of damping ratio in the structure. Also the FORTRAN computer programme for solving the Duhamel's Integral has been improved in this paper.
\end{abstract}

Keyword: Comparative studies; Damping ratio; Earthquake engineering design; Effective damping 